\title{
Optical Simulation of Top-Emission Organic Light Emitting Devices with Passivation Layer
}

\author{
Chia-Chiang Shiau ${ }^{* a}$, Hung-Chi Chen ${ }^{\mathrm{a}}$, Jiun-Haw Lee ${ }^{\mathrm{a}}$, Yean-Woei Kiang ${ }^{\mathrm{a}}$, C. C. Yang ${ }^{\mathrm{a}}$, and \\ F. H. Yang ${ }^{\mathrm{b}}$ \\ ${ }^{\mathrm{a}}$ Graduate Institute of Communication Engineering, Graduate Institute of Electro-Optical \\ Engineering, and Department of Electrical Engineering, National Taiwan University, No. 1, Sec. \\ 4, Roosevelt Road, Taipei, Taiwan, R.O.C.; \\ ${ }^{\mathrm{b}}$ AIXTRON Taiwan Corporation, Science-based Industrial Park, Hsin-Chu, Taiwan, R.O.C.
}

\begin{abstract}
In this paper, we report simulation results of a top-emission organic light-emitting device (TOLED) with a passivation layer composed of silicon dioxide $\left(\mathrm{SiO}_{2}\right)$ and silicon nitride $\left(\mathrm{Si}_{3} \mathrm{~N}_{4}\right)$ to protect organic layers from oxygen and moisture. Usually, the thickness of such a layer is about several micrometers. The electrode material of the device used for simulation is silver $(\mathrm{Ag})$. The anode is thick and opaque while the cathode is thin and semi-transparent. The structure is capped with three pair of $\mathrm{SiO}_{2}$ and $\mathrm{Si}_{3} \mathrm{~N}_{4}$ as the passivation layer. When $\mathrm{SiO}_{2}$ thickness is less than $2 \mu \mathrm{m}$, the output intensity changes and the spectrum peak shifts. The peaks oscillate as a function of the $\mathrm{SiO}_{2}$ thickness and the period is about $170 \mathrm{~nm}$. When the $\mathrm{SiO}_{2}$ thickness is over $2 \mu \mathrm{m}$, more than single peak are observed. With a simple model, we found that the frequency difference between two peaks corresponds to the free spectral range of the fabry-perot cavity formed by passivation layer. When a passivation layers is added on the TOLED, the microcavity effect results in the presence of multi-peaks. It limits the view angle and decreases the color purity.
\end{abstract}

Keywords: OLED, optical simualtion.

\section{INTRODUCTION}

OLED is one of the most promising candidates for the next generation display technologies since it exhibits advantages such as self emissive, wide-view-angle, flexible-substrate-capability and potentially low cost [1]. For recent years, OLED technologies also show a remarkable progress in material and device for improving the efficiency and the stability [2], [3]. Typically, such a device consists of an indium-tin-oxide (ITO) anode, a p-type and n-type organic layers, and a metal cathode. Electro-luminescence (EL) is generated from the organic layers and coupled out of the device from the ITO side. To drive devices in a display, passive matrix (PM) and active matrix (AM) techniques were used. Although the cost is lower, PM architecture require high peak drive currents to provide high peak luminance, which results in higher power consumption along with adverse effects on the OLED reliability. On the other hand, AM technique is more suitable for the large size, high quality and long lifetime applications. However, the thin-film transistor (TFT) on the AM backplane decreases the aperture ratio of a pixel that decreases the emission efficiency. Top-emission OLED (or TOLED) is one of the most promising technology for achieving high aperture ratio active matrix OLED. However, the fabrication process and the optical design of such a device is quite different from the conventional bottom-emission OLED. TOLED structure has strong microcavity effect because of the high reflective anode and semitransparent metal cathode. It causes the optical structure of TOLED more complex than traditional bottom emission OLED. Usually, TOLED is capped with a dielectric layer to enhance

*r92941057@ntu.edu.tw; phone 8862 2363-5251 ext.125; fax 8862 2367-7467 
the external quantum efficiency (EQE) [4], [5]. Besides, for achieving longer lifetime, passivation layer are necessary to protect organic materials and cathode from oxygen and moisture.

Usually, the thickness of the passivation layer is about several micrometers. It is composed with $\mathrm{SiO}_{\mathrm{N}}$ with a refractive index of 1.4. Typically, pairs of the inorganic layer (like $\mathrm{SiO}_{\mathrm{N}}$ ) and the organic (like some polymers) are fabricated to serve as the functions of passivation and stress relaxation, respectively. The structure usually needs three pairs to protect device from moisture and oxygen efficiently. On the other hand, because the EL from the TOLED passes through the passivation layer, the EL characteristics will be change by the passivation layer. Here, we use an electromagnetic theory model to simulation the optical characteristics of TOLED with passivation layer. Our results indicate that both the efficiency and the color spectra of TOLED vary strongly with the thickness and materials of the passivation layer. The variation in efficiency and color can be explained by fabry-perot cavity effects of thick passivation layer. The theory details are represented in section 2 . The results and the related discussions are shown in section 3. Conclusions are drawn in section 4.

\section{THEORY}

The TOLED device consists of multilayer structure as shown in Fig.1. The devices are fabricated on glass substrate with an opaque anode Ag. The organic multilayer structure consists of $60 \mathrm{~nm}$ N,N'-dipheny1-N, $\mathrm{N}^{\prime}$-bis(1,1'-bipheny1)-4,4'-damine (NPB) as the hole-transporting layer (HTL), 60nm tris-(8-hydroxyquinoline) aluminum (Alq3) as the electron-transporting layer (ETL) and emitting layer (EML). The $\mathrm{EL}$ is observed through a semitransparent metal cathode consists of $20 \mathrm{~nm}$ Ag. Finally, a dielectric layer such as $\mathrm{SiO}_{2}$ was added to enhance the output coupling efficiency. To understand the physical mechanisms of the radiation from an OLED, we need first to derive the governing equations for electric and magnetic field in each layer of the device. We assume the electrons and holes recombine and form excitons at the HTL and ETL interface. The excitons in the emission layer can be represented as oscillating dipoles. For calculating the radiation from dipole sources embedded in the emission layer (EML), we first introduce the electric Hertzian vector potential due to a vertical or horizontal dipole embedded in the EML[6], [7], [8] , [9]. Then, with $e^{j \omega t}$ time-harmonic dependence, the electric and magnetic fields can be derived from the Hertzain vector potential.

$$
\begin{aligned}
& E=k_{0}{ }^{2} n^{2} \Pi+\nabla \nabla \cdot \Pi \\
& H=-j \omega \varepsilon_{0} n^{2} \nabla \times \Pi
\end{aligned}
$$

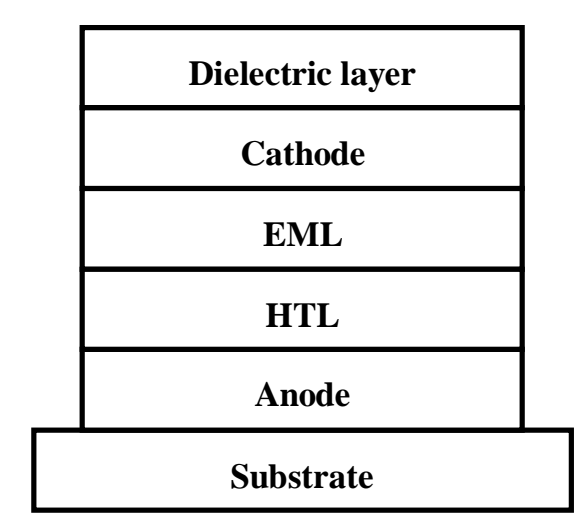

FIG.1. Device structure of Top-emission OLED. 
where $\mathrm{n}$ is the complex refractive index and $k_{0}=\omega \sqrt{\mu_{0} \varepsilon_{0}}=2 \pi / \lambda$ is the wave number or propagation constant in vacuum. The wave equations for $\Pi$ can be expressed as

$$
\begin{aligned}
& \left(\nabla^{2}+n_{i}^{2} k_{0}^{2}\right) \Pi=0, \text { in } i^{\text {th }}(\text { non-emission) layer } \\
& \left(\nabla^{2}+n_{e}{ }^{2} k_{0}^{2}\right) \Pi=\frac{-p \delta\left(r-r^{\prime}\right)}{\varepsilon_{0} n_{e}{ }^{2}}, \text { in EML }
\end{aligned}
$$

where $p$ denotes a dipole source which is located at $r=r^{-}$and $n_{e}$ is the wavelength-dependent complex refractive index for the EML. We employ the property of axial symmetry and take the Fourier-Bessel transform to obtain an integral expression for the Herzian vector potential in each layer of OLED. By using the boundary conditions that the tangential electric and magnetic fields are continuous across the interface between layers, we can determine the unknown coefficients in the integral expression of Hertzian potential from which all the electromagnetic field components can then be calculated accordingly. To calculate the radiation field in the far region, the saddle-point method (as shown in Fig. 2(a)) is usually used to perform the integration over the radial wavenumber [10]. If the outside layer is very thick, such as glass substrate in bottom emission OLED, we safely employ the saddle-point method to determine the mathematical expression of the radiation field $\mathrm{E}$ at the original interface between the air region and the outside layer (as shown in Fig. 2(b)). Then the radiation field E, acting as an incident field in the glass layer, will propagate in thick layer subjected to multiple reflections and transmissions. Finally, we can determine the effective transmission coefficient for $\mathrm{E}$ to transmit to the air region. Note that the radiated field in the thick outside layer $\mathrm{E}$ is not a plane wave but a spreading beam wave. Using the principle of energy conservation and Snell's law, we can derive a multiplicative spread factor.

$$
S=\frac{n_{\text {air }}}{n_{\text {glass }}}\left[\frac{\cos \theta_{\text {air }}}{\sqrt{1-\left(\frac{n_{\text {air }}}{n_{\text {glass }}} \sin \theta_{\text {air }}\right)^{2}}}\right]^{\frac{1}{2}}
$$

where $\Theta_{\text {air }}$ is the refractive angle from the glass layer to the air region. The above procedure is applicable to either a
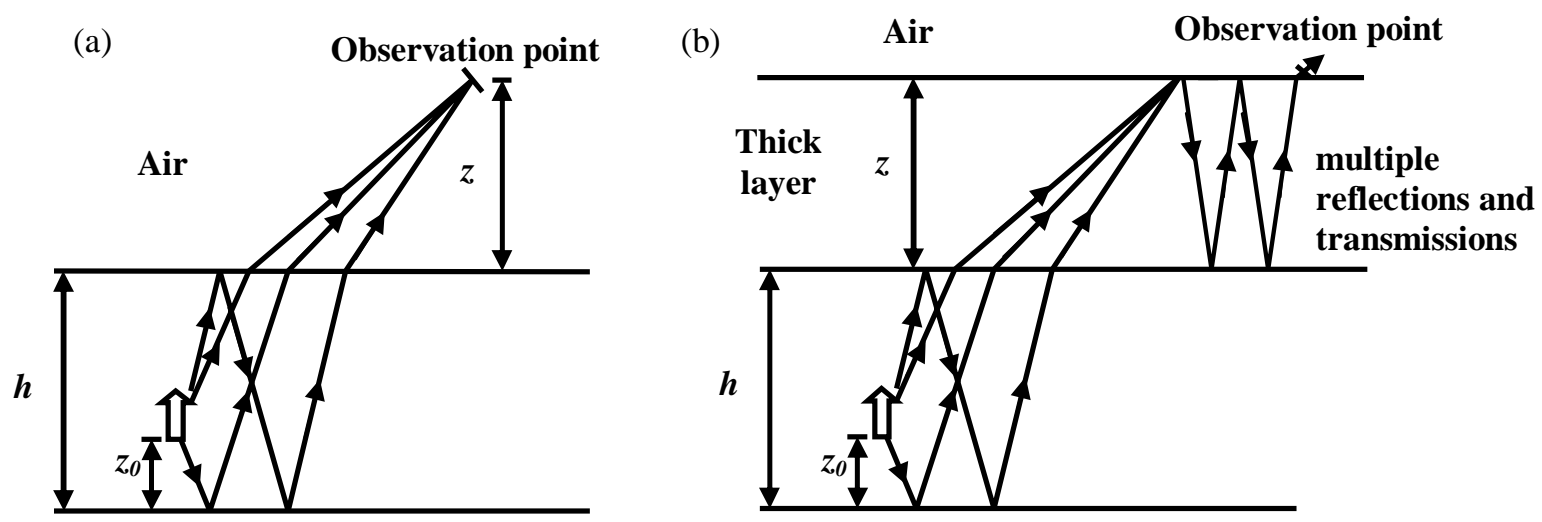

FIG.2. The Saddle-point method. 
vertical dipole source or a horizontal one. The outputs of this simulation program include the radiant intensity pattern due to randomly oriented dipoles, and the electroluminescence emission patterns for a given photoluminescence.

For a dipole source operating at a single frequency, strong interference of the coherent fields reflected from interfaces between layers results in a rapidly oscillatory radiation pattern. In fact, every real light source should have a finite bandwidth and the interference will be smoothened. Therefore, we assume that the dipole source is of a finite bandwidth (say, $0.5 \mathrm{~nm}$ ), and the resultant radiation field is obtained by coherently averaging the fields over that range of wavelengths.

\section{RESULTS AND DISCUSSIONS}

As shown in Fig. 1, a TOLED was deposited with a dielectric layer on top of a semitransparent metal cathode to enhance the output coupling effect. The dependence of the EL intensity emitted in forward direction on the dielectric thickness is illustrated in Fig. 3. The peak of EL spectrum first increases and red-shifts, then blue-shift with increasing the dielectric layer thickness. Final, it recovers to the original spectrum and intensity. The EL spectrum changes with dielectric thickness that has a period of 170nm. From Fig. 4, we can see that the different refractive index material cause different enhance effect and have different peak position. The higher refractive index material has larger oscillating amplitude and shorter period.

Fig. 5 illustrate the EL intensity of TOLED when use different Alq3 thickness. With increasing the thickness of Alq3 layer, the relative intensity of the same dielectric layer decreases, and the peak position shifts. In fact, this can be explained by using the planar microcavity effects.

$\left|E_{\text {out }}^{u p}\right|^{2}=\left|E_{\text {in }}\right|^{2} \times \frac{T_{e f f} \times\left|1+r_{e f f} e^{2 i \Phi}\right|^{2}}{\left|1-r_{1} r_{e f f} e^{2 i q}\right|^{2}}$

where $\left|E_{\text {out }}^{u p}\right|^{2}$ is the output intensity, $\left|E_{\text {in }}\right|^{2}$ is the intensity of emission dipoles, $\mathrm{r}_{1}$ is the reflectivity of NPB to

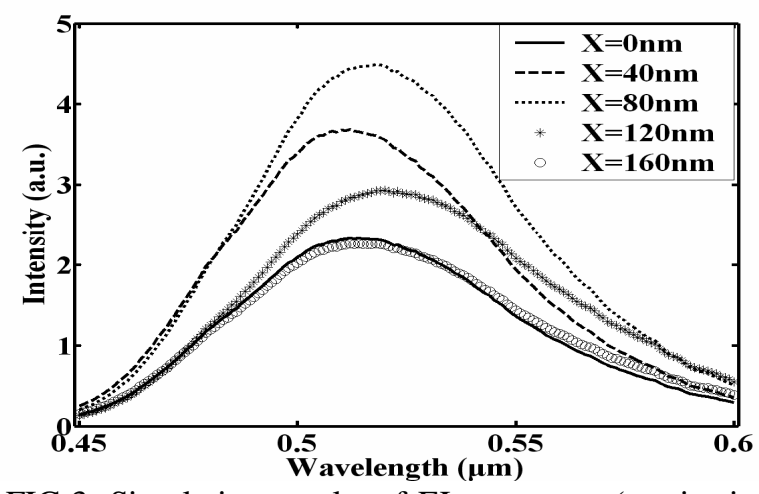

FIG.3. Simulation results of EL spectrum (at viewing angle $=0^{\circ}$ ) various dielectric layer thickness. OLED structure: $\mathrm{Ag} / 60 \mathrm{~nm} \mathrm{NPB} / 60 \mathrm{~nm} \mathrm{Alq}_{3} / 20 \mathrm{~nm} \mathrm{Ag} / \mathrm{Xnm}$ dielectric layer.

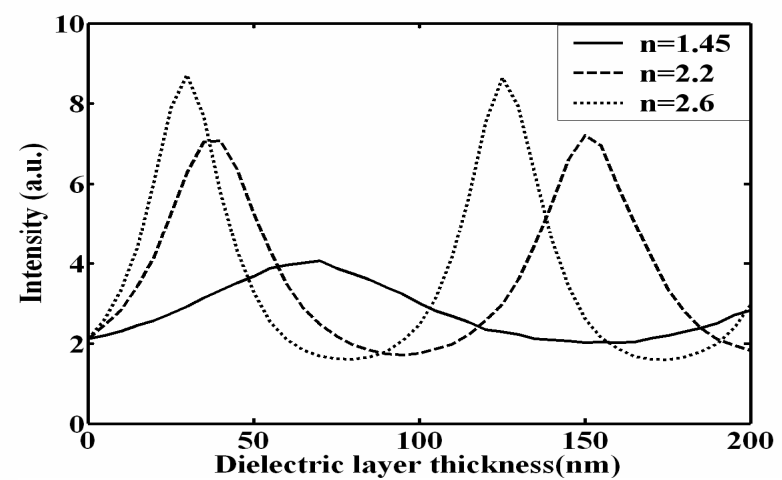

FIG.4. Simulation results of intensity (at wavelength $=500 \mathrm{~nm}$, viewing angle $=0^{\circ}$ ) as a function of different dielectric layer refractive indices and different dielectric layer thickness. OLED structure: $\mathrm{Ag} / 60 \mathrm{~nm}$ $\mathrm{NPB} / 60 \mathrm{~nm} \mathrm{Alq}_{3} / 20 \mathrm{~nm} \mathrm{Ag} / \mathrm{Xnm}$ dielectric layer. 
anode, $\mathrm{r}_{\text {eff }}$ and $\mathrm{T}_{\text {eff }}$ are the effective reflectivity and transmission of $\mathrm{Alq}_{3}$ pass through the cathode and dielectric layer to the air, respectively. The symbol $\varphi$ is the optical length of total organic layer, and $\Phi$ is the optical length between the dipoles and anode. The out intensity is affected by the total organic layer thickness and the position of the emission dipoles. We can see that the periods in Fig. 5 are not affected by the thickness of the organic layer. It is determined by the dielectric material. The output intensity of the three cases in Fig.5 is different. With increasing the thickness of the EML, the intensity decreases since the denominator of equation (6) is smaller in a thinner device within our simulation range.

However, for a real application, a passivation layer is needed to protect the device from the ingression of water and oxygen. Typically, a passivation layer is composed with pairs of the inorganic layer (like $\mathrm{SiO}_{\mathrm{N}}$ ) and the organic (like some polymers) to serve as the functions of passivation and stress relaxation, respectively. However, due to the limitation of layer numbers in our simulation program, we regard the passivation layers as one single thick layer with an effective refractive index when the thickness of such a layer exceed ten times of the wavelength. With a simplified device structure, we found that the simulation results are quite similar between the case of using a single thick and multiple pair passivation layers with the same optical length. Such results will be published elsewhere.

Typically, passivation layer over the top-emission OLED is several micrometers in thickness. Fig. 6 illustrates the output EL when the passivation layer is about several micrometers. With increasing the dielectric layer thickness, the more peaks appears and the spacing among peaks become closer. The spectrum of such a device exhibits multiple peaks which correspond to the free spectral range of the fabry-perot cavity formed by this layer. It can be described as $\Delta \mathrm{f}=\mathrm{c} / 2 \mathrm{~nL}$ where $\mathrm{c}$ is the velocity of light in vacuum, $\mathrm{n}$ is the refractive index of $\mathrm{SiO}_{2}$, and $\mathrm{L}$ is the thickness of $\mathrm{SiO}_{2}$. The microcavity effect results in the presence of multi-peaks. It limits the view angle and decreases the color purity. Fig. 7 illustrates EL spectrum when the passivation compound of $\mathrm{SiO}_{2}$ and several different materials. It shows that when the refractive index difference between the two materials is larger, the amplitude oscillation of the EL spectrum is bigger. This is a serious problem with three pair of this stack structure for passivation layer. It will change the top-emission OLED EL spectrum different when adding the passivation layer.

In order to overcome the oscillation problem, we increase the thickness of the passivation layer. The spacing among peaks become closer and it will become indistinguishable when the thickness is over $200 \mu \mathrm{m}$. Fig. 8 show the simulation results of output EL spectrum (at viewing angle $=0^{\circ}$ ) of OLED device with different passivation layer structure. Device 1 is the TOLED without any dielectric or passivation layer. Device 2 is the TOLED with $200 \mu \mathrm{m}$

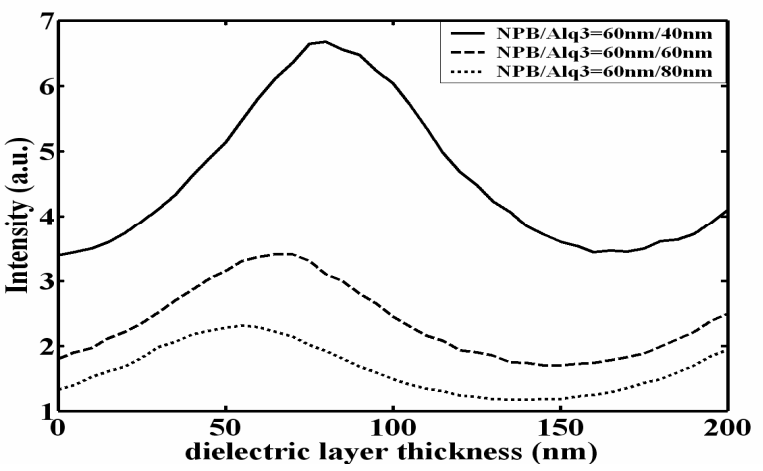

FIG.5. Simulation results of intensity (at wavelength $=500 \mathrm{~nm}$, viewing angle $=0^{\circ}$ ) as a function of different dielectric layer thickness when use different $\mathrm{Alq}_{3}$ thickness. OLED structure: $\mathrm{Ag} /$ $60 \mathrm{~nm} \mathrm{NPB} / \mathrm{Alq}_{3} / 20 \mathrm{~nm} \mathrm{Ag} / \mathrm{Xnm}$ dielectric layer (refractive index $=1.45$ ).

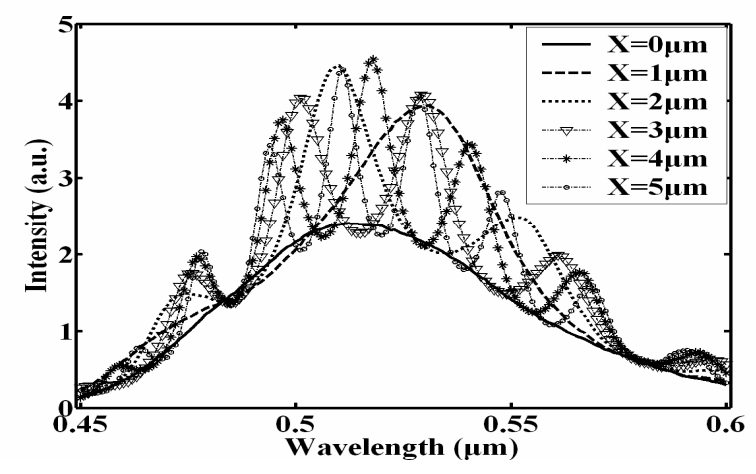

FIG.6. Simulation results of intensity (at viewing angle $=0^{\circ}$ ) as a function of different passivation layer thickness. OLED structure: Ag/ 60nm NPB/ 60nm $\mathrm{Alq}_{3} / 20 \mathrm{~nm} \mathrm{Ag} / \mathrm{X} \mu \mathrm{m}$ passivation layer( refractive index=1.45). 


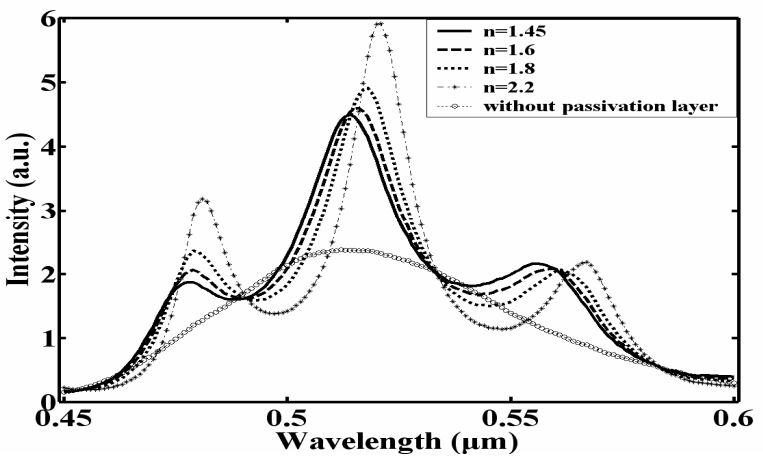

FIG.7. Simulation results of intensity (at viewing angle $=0^{\circ}$ ) as a function of different passivation layer refractive index. OLED structure: $\mathrm{Ag} / 60 \mathrm{~nm} \mathrm{NPB} /$ $60 \mathrm{~nm} \mathrm{Alq} / 20 \mathrm{~nm} \mathrm{Ag} / 2 \mu \mathrm{m} \mathrm{SiO}_{2}$ ( refractive index = $1.45) / 200 \mathrm{~nm}$ difference material (refractive index $n$ ).

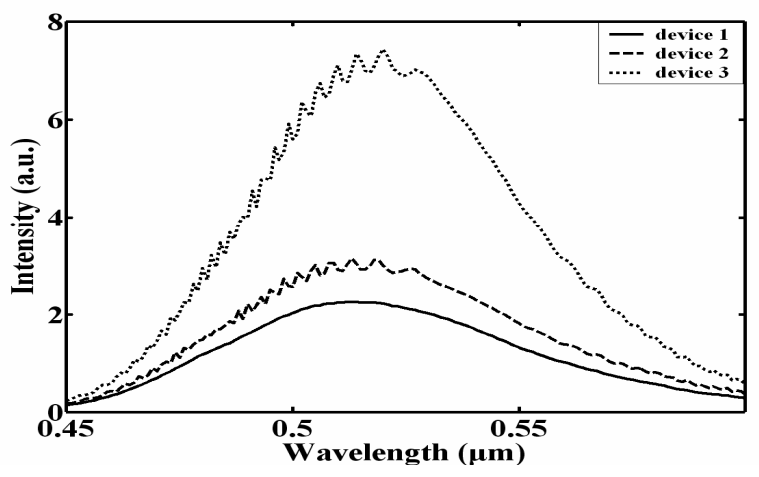

FIG. 8. Simulation results of output EL spectrum of device with different passivation layer structure. TOLED structure: Ag/ 60nm NPB/ 60nm Alq $/$ $20 \mathrm{~nm} \mathrm{Ag/} \mathrm{passivation} \mathrm{layer.} \mathrm{Device} \mathrm{1:} \mathrm{without}$ passivation. Device 2: with $200 \mu \mathrm{m}$ passivation layer. Device 3: with 35nm dielectric layer/ 200 $\mu \mathrm{m}$ passivation layer.

passivation layer (refractive index $=1.45$ ). In device 3, we add a dielectric layer (refractive index $=2.6$ ) between the cathode and the passivaition to enhance the output efficiency. With simulation results, when the dielectric layer is $35 \mathrm{~nm}$, we can obtain the highest output efficiency. We can see that the device without passivation layer (device 1) has lowest output intensity. Adding a passivation layer on the TOLED device can enhance the efficiency and the EL spectrum does not change obviously (see device 2). Besides, we can add a dielectric layer between the cathode and the passivation layer to enhance the output coupling efficiency.

\begin{tabular}{|c|c|c|c|c|c|c|c|c|}
\hline \multirow{2}{*}{$\begin{array}{c}\text { Viewing } \\
\text { angle }\end{array}$} & \multirow{2}{*}{$\begin{array}{c}\text { CIE } \\
\text { coordinate }\end{array}$} & Device & Device & \multicolumn{3}{|c|}{ Device 3 with different dielectric layer thickness } \\
\cline { 3 - 9 } & & 1 & 2 & $20 \mathrm{~nm}$ & $25 \mathrm{~nm}$ & $30 \mathrm{~nm}$ & $35 \mathrm{~nm}$ & $40 \mathrm{~nm}$ \\
\hline \multirow{2}{*}{$0^{\circ}$} & $\mathrm{X}$ & 0.2137 & 0.2171 & 0.1937 & 0.1943 & 0.2003 & 0.2117 & 0.2268 \\
\cline { 2 - 9 } & $\mathrm{Y}$ & 0.5608 & 0.5662 & 0.5562 & 0.5668 & 0.5828 & 0.5981 & 0.6077 \\
\hline \multirow{2}{*}{$30^{\circ}$} & $\mathrm{X}$ & 0.2147 & 0.2157 & 0.1863 & 0.1830 & 0.1839 & 0.1896 & 0.1996 \\
\cline { 2 - 9 } & $\mathrm{Y}$ & 0.5625 & 0.5675 & 0.5467 & 0.5520 & 0.5657 & 0.5846 & 0.6020 \\
\hline \multirow{2}{*}{$60^{\circ}$} & $\mathrm{X}$ & 0.2183 & 0.1969 & 0.1741 & 0.1688 & 0.1647 & 0.1618 & 0.1592 \\
\cline { 2 - 9 } & $\mathrm{Y}$ & 0.5722 & 0.5639 & 0.5143 & 0.5019 & 0.4908 & 0.4829 & 0.4777 \\
\hline
\end{tabular}

Table I. The coordinates of different passivation layer structure and different viewing angle.

Table I show the CIE coordinate of different passivation layer structure in Fig. 8. The CIE coordinate of device 1 and device 2 are almost the same except at viewing angle $=60^{\circ}$ has a little blue shift. When we add a dielectric layer between the cathode and the passivation layer, it cause serious CIE shift especially at large viewing angle, although higher out coupling efficiency is achieved.

\section{SUMMARY}

In this paper, we have shown the simulation results of a TOLED with a passivation layer. Such a layer is typically 
several micrometers in thickness. The spectrum of such a device exhibits multiple peaks which correspond to the free spectral range of the fabry-perot cavity formed by this layer. The microcavity effect may result in the presence of multi-peaks. It limits the view angle and decreases the color purity. With increasing the thickness of the passivation layer, the spacing among peaks become closer and it will become indistinguishable when the thickness is over $200 \mu \mathrm{m}$, and the CIE coordinate does not change much. Besides, we can add a dielectric layer between the cathode and passivation layer to enhance to out coupling efficiency, but it will cause the EL spectrum shift.

\section{REFERENCES}

1. C. W. Tang and S. A. Vanslyke, Appl. Phys. Lett. 51, 913 (1987).

2. C. F. Madigan, M. H. Lu, and J. C. Sturm, Appl. Phys. Lett. 761650 (2000)

3. S. Möller and S. R. Forrest, J. Appl. Phys 913324 (2003)

4. H. Riel, S. Karg, T. Beierlein, W. Rieß, and K. Neyts, J. Appl. Phys 945290 (2003)

5. H. Riel, S. Karg, T. Beierlein, B. Ruhstaller, and W. Rieß, Appl. Phys. Lett. 82466 (2003)

6. J. A. E. Wasey and W.L. Barnes, J. Modern Optics. 47, 725 (2000)

7. $\quad$ K. B. Kahen, Appl. Phys. Lett. 781649 (2001)

8. J. R. Wait, IEEE Trans. On Antennas and Propagation 15645 (1967)

9. A. W. Biggs and H. Myron Swarm, IEEE Trans. On Antennas and Propagation 16201 (1968)

10. A. Ishimaru, "Electromagnetic Wave Propagation, Radiation, and Scattering" London: Prentice-Hall International (1991) 